\title{
Consideraciones Anatomo-Quirúrgicas del Ligamento Reflejo
}

\author{
Reflected Ligament: Anatomical and Surgical Considerations
}

\author{
Rubén Daniel Algieri, ${ }^{* * *}$; Eduardo Pro**** María Soledad Ferrante ${ }^{* * * * *}$; \\ Juan Sebastián Ugartemendía ${ }^{* * * * *}$ \& Maria de las Mercedes Bernadou*****
}

\begin{abstract}
ALGIERI, R. D.; PRO, E.; FERRANTE, M. S.; UGARTEMENDÍA, J. S. \& BERNADOU, M. M. Consideraciones anatomoquirúrgicas del ligamento reflejo. Int. J. Morphol., 33(4):1361-1364, 2015.

RESUMEN: La anatomía de la región inguinal está bien descrita y detallada en la literatura, pero existen algunas controversias vinculadas al ligamento reflejo y a su inserción medial. El objetivo fue analizar la inserción del ligamento inguinal reflejo del músculo oblicuo externo en la línea alba, mediante lo observado en disecciones cadavéricas. Se utilizaron 30 cadáveres formolizados, en los que el ligamento reflejo fue disecado hasta su inserción en la línea alba. Se encontró la presencia del ligamento inguinal reflejo en 25 $(83,33 \%)$ casos, $16(64 \%)$ hombres y $9(36 \%)$ mujeres, de los cuales sólo en 2 (8\%) casos se observó la inserción interdigitada del ligamento inguinal reflejo con el contralateral; y en 5 (16,67\%) casos (3 mujeres y 2 hombres), se constató ausencia del mencionado ligamento. El ligamento reflejo fue identificado en la mayoría de nuestros ejemplares. Este ligamento contribuye a la formación de la pared posteromedial del anillo inguinal superficial, siendo importante la necesidad de su conocimiento anatómico para su aplicación en la reparación quirúrgica de hernias inguinales con colocación protésica por vía anterior.
\end{abstract}

PALABRAS CLAVE: Ligamento inguinal reflejo; Región inguinal; Músculo oblicuo externo; Línea alba.

\section{INTRODUCCIÓN}

La anatomía de la región inguinal está bien descrita y detallada en la literatura, pero existen algunas controversias vinculadas al ligamento reflejo y a su inserción medial (Fig. 1). Así, este ligamento es motivo de discusión muchas veces entre los anatomistas que lo consideran proveniente del lado opuesto y que atraviesa la línea media formando uno de los limites, llamado pilar posterior, del orificio inguinal superficial (Barroetaveña et al., 2013; Carbonell Tatay, 2001; Casiraghi et al., 1969; Latarjet \& Ruiz Liard, 2005; Moore et al., 2013; Ferraina \& Oria, 2002; Rouvière \& Delmas, 2005; Testut \& Jacob, 1964; Testut \& Latarjet, 1984) y entre los que lo consideran verdaderamente como un ligamento reflejo proveniente del mismo lado y terminando en la línea alba (Lytle, 1974, 1979; Nyhus \& Condon, 1991; Tubbs et al., 2009; Pró, 2014), otros en cambio hacen su descripción sin identificarlo (Drake et al., 2005).

El objetivo fue analizar la inserción del ligamento inguinal reflejo del músculo oblicuo externo en la línea alba, mediante lo observado en disecciones cadavéricas.

\section{MATERIAL Y MÉTODO}

Se utilizaron 30 cadáveres adultos formalizados al 10\% de la III Cátedra de Anatomía de la Facultad de Medicina de la Universidad de Buenos Aires, Argentina, 18 hombes y 12 mujeres, en los cuales se disecó la pared anterior del abdomen hasta observar los 3 pilares de inserción del músculo oblicuo externo en el pubis, tanto del lado derecho como izquierdo. Se identificó el ligamento inguinal reflejo hasta su inserción en la línea alba siguiendo la dirección de sus fibras sin seccionarlo.

\section{RESULTADOS}

De los 30 cadáveres, en 25 casos (83,33\%), correspondientes a 16 hombres (64\%) y 9 mujeres (36\%), se encontró la presencia del ligamento inguinal reflejo. De ellos, sólo en 2 casos (8\%) se observó la inserción interdigitada del ligamento inguinal reflejo con el contralateral (Fig. 2); y

\footnotetext{
Especialista en Cirugía General, Jefe de Servicio de Cirugía General del Hospital Aeronáutico Central, Buenos Aires, Argentina.

** Prof. Regular Adjunto de Anatomía, Docente Adscripto en Cirugía, Facultad de Medicina, Universidad de Buenos Aires, Buenos Aires, Argentina.

*** Profesor Titular de la Segunda Cátedra de Anatomía, Facultad de Medicina, Universidad de Buenos Aires, Buenos Aires, Argentina.

***** Especialista en Cirugía General. Instructora de Residentes de Cirugía General del Hospital Aeronáutico Central. Jefe de Trabajos Prácticos de Anatomía, Facultad de Medicina, Universidad de Buenos Aires, Buenos Aires, Argentina.

******* Residente de Cirugía General del Hospital Aeronáutico Central, Buenos Aires, Argentina.
} 


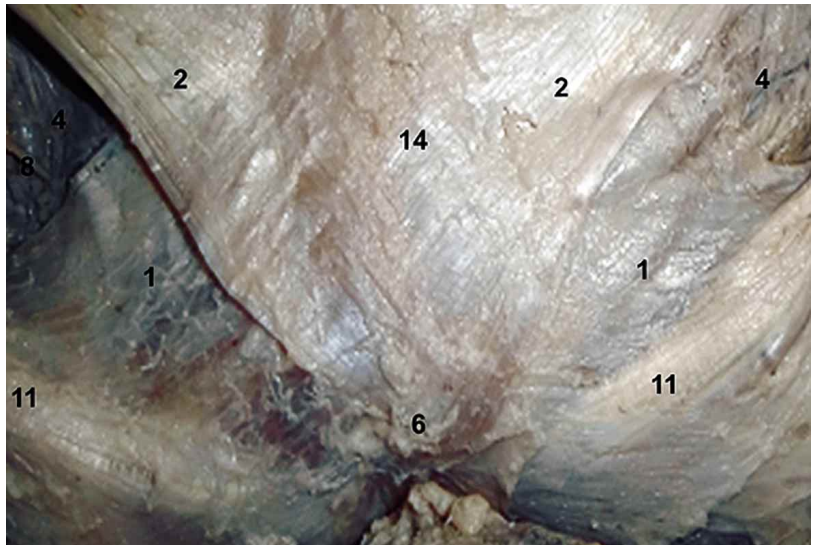

Fig. 1. Región inguinal bilateral donde se observan ambos ligamentos reflejos. 1) Ligamento reflejo; 2) Pilar medial; 4) Fascia Transversalis; 6) Pubis; 8) Nervio iliohipogastrico; 11) Ligamento inguinal y 14) Línea alba.

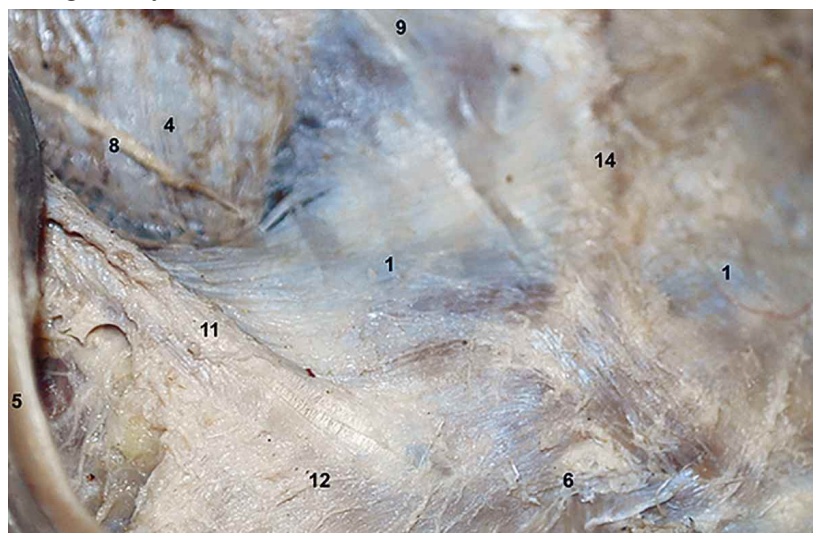

Fig. 2. Inserción del ligamento reflejo en la línea alba interdigitada con el contralateral. 1) Ligamento reflejo; 4) Fascia Transversalis; 5) Cordón espermático; 6) Pubis; 8) Nervio iliohipogastrico; 9) Tendón conjunto; 11) Ligamento inguinal; 12) Ligamento lacunar y 14) Línea alba.

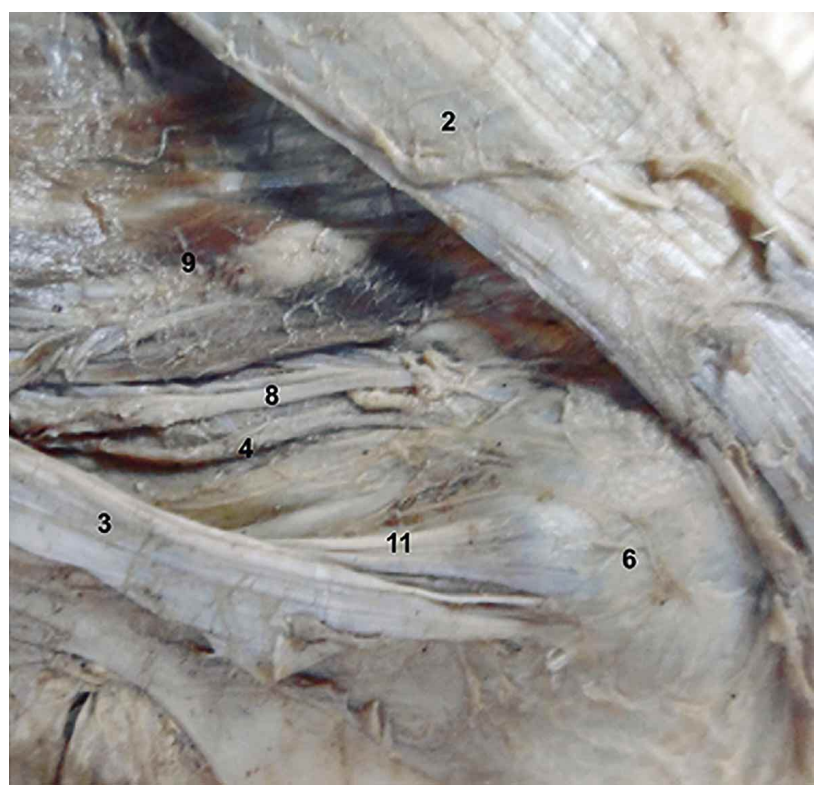

en 5 casos $(16,67 \%)$, correspondientes a 3 mujeres (60\%) y 2 hombres (40\%), se constató ausencia del mencionado ligamento (Fig. 3). No hubo estadísticamente diferencia significativa entre los lados derecho o izquierdo o entre ambos sexos.

La forma y el tamaño de los ligamentos inguinales reflejos fueron variables, pero en general, guardaban la forma triangular. En la mayoría, el ligamento reflejo se encontró extendiéndose desde el ligamento lacunar y el ligamento inguinal dirigiéndose oblicuamente hacia la línea media en un ángulo aproximado de $45^{\circ}$ para insertarse en la línea alba (Figs. 4 y 5 ).

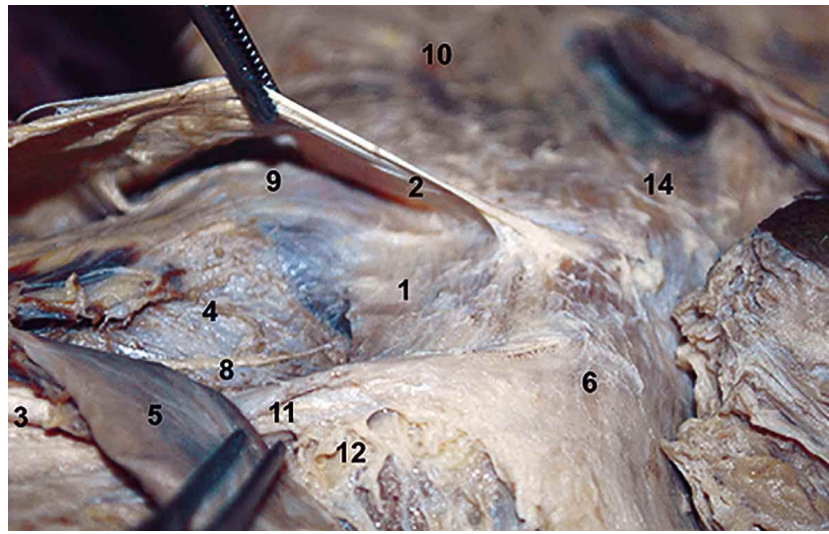

Fig. 4. Disección del conducto inguinal derecho. 1) Ligamento reflejo; 2) Pilar medial; 3) Pilar lateral; 4) Fascia Transversalis; 5) Cordón espermático; 6) Pubis; 8) Nervio iliohipogastrico; 9) Tendón conjunto; 10) Aponeurosis del músculo oblicuo externo; 11) Ligamento inguinal; 12) Ligamento lacunar y 14) Línea alba.

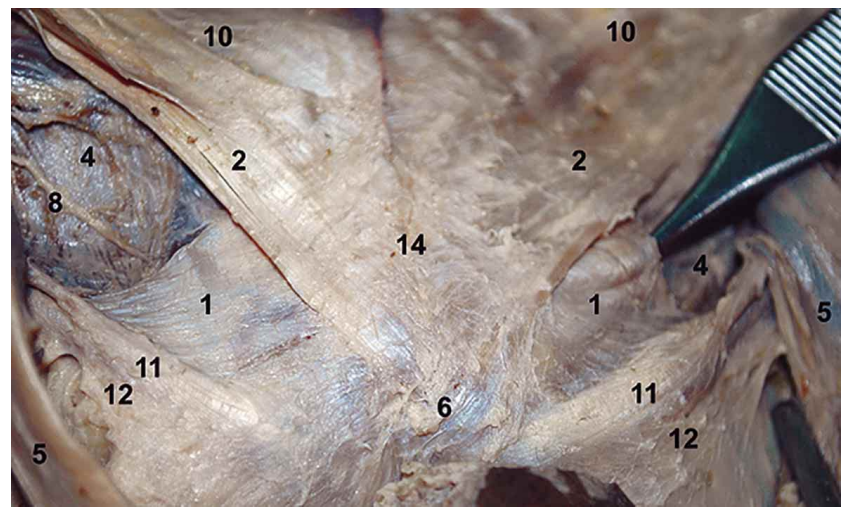

Fig. 5. Ligamento reflejo reparado. 1) Ligamento reflejo; 2) Pilar medial; 4) Fascia Transversalis; 5) Cordón espermático; 6) Pubis; 8) Nervio iliohipogastrico; 10) Aponeurosis del músculo oblicuo externo; 11) Ligamento inguinal; 12) Ligamento lacunar y 14) Línea alba.

Fig. 3. Ausencia del ligamento reflejo. 2) Pilar medial; 3) Pilar lateral; 4) Fascia Transversalis; 6) Pubis; 8) Nervio iliohipogastrico; 9) Tendón conjunto y 11) Ligamento inguinal. 


\section{DISCUSIÓN}

La tasa de incidencia de este ligamento varía según los autores, que oscilan entre el 1 al 3\% de la población en general (Tubbs et al.). El origen de estas fibras ha sido muy discutido. La mayoría de los autores asegura que provienen de la porción aponeurótica del músculo oblicuo externo del lado contralateral, que atravesando la línea media pasan por detrás del pilar interno y llegan al borde inferior del anillo inguinal superficial y cara ventral del tendón conjunto, es lo que los autores clásicos franceses llamaron "pilar posterior" o "ligamento de Colles" (Carbonell Tatay; Casiraghi et al.; Latarjet \& Ruiz Liard; Ferraina \& Oria; Rouvière \& Delmas; Testut \& Latarjet).

También ha sido descrito como un ligamento triangular formado a partir de una expansión del ligamento lacunar al unirse a la línea alba. Otros afirman que este ligamento deriva del límite inferior del anillo superficial y del ligamento lacunar, o proveniente del tendón conjunto (Lytle, 1974, 1979; Nyhus \& Condon; Tubbs et al.; Pró; Testut \& Jacob). Hay autores que refieren no haberlo encontrado en sus disecciones e incluso hay otros que ni lo mencionan en sus descripciones (Barroetaveña et al.; Drake et al.).

Como se observa, son distintas las posturas que hay entre los autores de acuerdo al origen y disposición de las fibras que corresponden a la pared posteromedial del anillo inguinal superficial, denominado ligamento reflejo.

Anatómicamente la aponeurosis del músculo oblicuo externo termina en una línea tendida desde el apéndice xifoides hasta la sínfisis pubiana pasando por la cara anterior del recto del abdomen, se entrecruza con la del lado opuesto en la línea media, siendo parte de la formación de la línea alba, y abajo en una cinta fibrosa que va desde el tubérculo del pubis (espina del pubis) hasta la espina iliaca anterosuperior formando el ligamento inguinal, que corresponde al piso del conducto inguinal. El labio posterior de este ligamento se extiende hacia arriba por una lámina fibrosa llamada tracto iliopúbico (cintilla iliopubiana de Thompson), esta cintilla sigue al ligamento inguinal y se extiende hasta la línea alba pasando por detrás del músculo recto mayor. La aponeurosis de inserción del músculo oblicuo externo interviene en la formación de la hoja anterior de la vaina del músculo recto del abdomen. También forma la pared anterior del conducto inguinal. Las inserciones púbicas de la aponeurosis del músculo oblicuo externo se hacen por fascículos fibrosos que forman los denominados pilares del anillo inguinal superficial, el ángulo de separación de estos pilares delimita dicho anillo. El límite externo de este orificio es el pilar lateral, lámina oblicua de aproximadamente $5 \mathrm{~mm}$ de ancho. Las fibras que pasan mediales al anillo forman el pilar medial. Estas fibras se extienden pasando delante de la vaina del músculo recto del abdomen, se entrecruzan en la línea media con las del lado contralateral (Testut \& Latarjet). Sobre el ángulo lateral de intersección de los pilares se hallan un grupo de fibras arciformes que cubren dicho ángulo llamadas fibras intercrurales (arciformes de Nicaise) (Fig. 6), que son fibras de refuerzo de la aponeurosis del músculo oblicuo externo extendidas entre los pilares lateral y medial. El ligamento inguinal se divide en tres porciones: lateral, por delante del músculo iliopsoas, se inserta por medio de dos haces en la fascia lata, entre los cuales pasa el nervio cutáneo femoral lateral, el resto termina en la fascia ilíaca; intermedia, donde

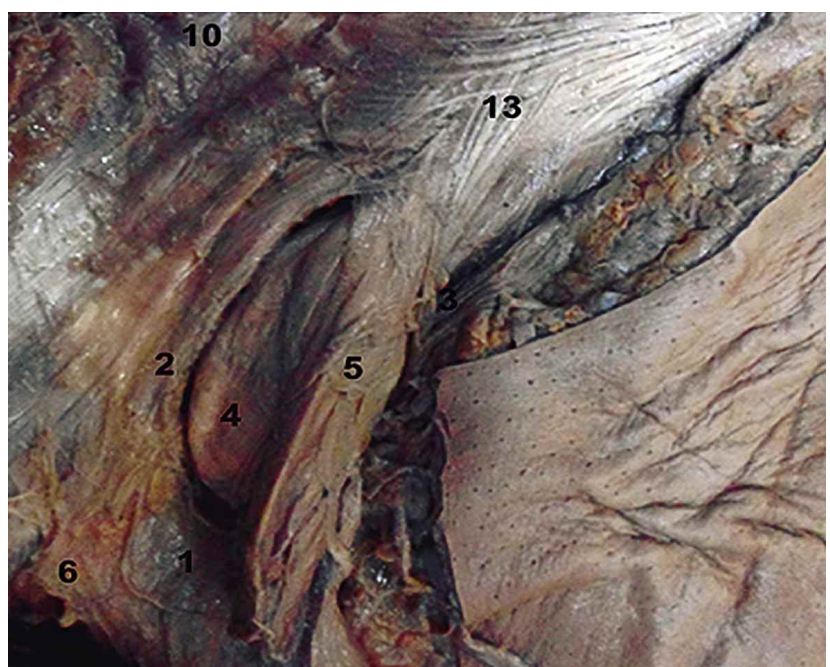

Fig. 6. Fibras intercrurales. 1) Ligamento reflejo; 2) Pilar medial; 3) Pilar lateral; 4) Fascia Transversalis; 5) Cordón espermático; 6) Pubis; 10) Aponeurosis del músculo oblicuo externo y 13) Fibras intercrurales.

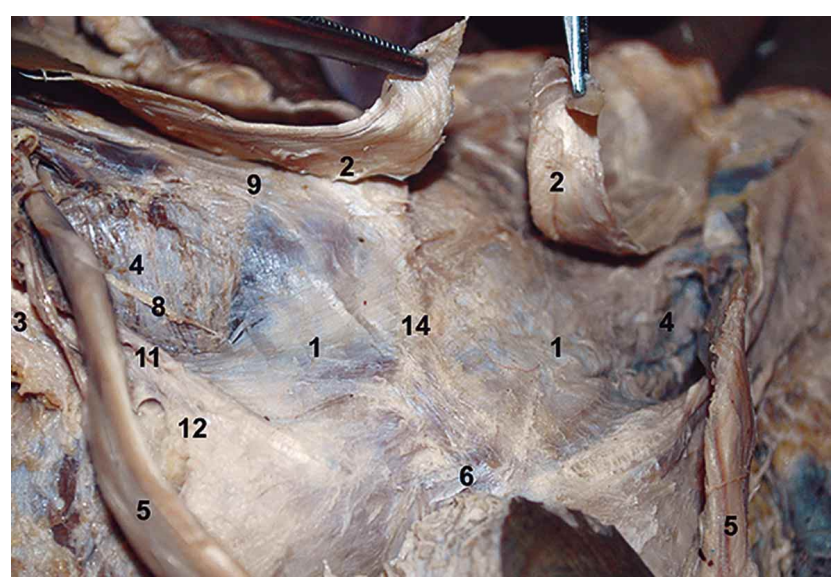

Fig. 7. Pilares mediales seccionados, se observan las fibras del ligamento reflejo y su inserción en la línea alba. 1) Ligamento reflejo; 2) Pilar medial; 3) Pilar lateral; 4) Fascia Transversalis; 5) Cordón espermático; 6) Pubis; 8) Nervio iliohipogastrico; 9) Tendón conjunto; 11) Ligamento inguinal; 12) Ligamento lacunar y 14) Línea alba. 
se encuentra el anillo femoral, por donde pasan los vasos femorales; y medial, pertenece al ligamento lacunar y al ligamento reflejo. Entre las porciones lateral e intermedia se despliega el arco iliopectíneo, que va desde el ligamento inguinal a la eminencia iliopúbica separando la laguna muscular de la laguna vascular. El ligamento lacunar (de Gimbernat) son fibras de la aponeurosis del músculo oblicuo externo que se reflejan hacia el pubis antes de llegar al tubérculo del pubis, se inclinan hacia abajo, atrás y afuera y se insertan en el pecten del pubis (cresta pectínea), formando una lámina fibrosa triangular (Casiraghi et al.; Moore et al.). El ligamento reflejo (de Colles) es un desdoblamiento de las fibras de inserción medial del ligamento inguinal y lacunar del mismo lado que se proyectan hacia arriba pasando por detrás del anillo inguinal superficial, dirigiéndose oblicuamente hacia la línea media para insertarse en la línea alba y contribuye a la formación de la pared posteromedial del anillo inguinal superficial (Nyhus \& Condon, Pró) (Fig. 7).

\section{CONCLUSIONES}

El ligamento inguinal reflejo, identificado en la mayoría de nuestros ejemplares, se extiende desde los ligamentos lacunar e inguinal del mismo lado para terminar a nivel medial en la línea alba y contribuye a la formación de la pared posteromedial del anillo inguinal superficial, siendo importante la necesidad de su conocimiento anatómico para su aplicación en la reparación quirúrgica de hernias inguinales con colocación protésica por vía anterior.

ALGIERI, R. D.; PRO, E.; FERRANTE, M. S.; UGARTEMENDÍA, J. S. \& BERNADOU, M. M. Reflected ligament: Anatomical and surgical considerations. Int. J. Morphol., 33(4):1361-1364, 2015.

SUMMARY: Anatomy of the inguinal region is well described in literature, but there is lack of information related to the reflected ligament and a dichotomy regarding its medial insertion. The aim was to carry out an observational analysis, through dissections, of the insertion of the reflected ligament of the external oblique muscle at the linea alba. We used 30 formolized corpses were, on which the reflected ligament was dissected to its insertion into the linea alba. Of the 30 bodies, we found the reflected ligament in $25(83.33 \%)$, being 16 male $(64 \%)$ and 9 females (36\%), but only in 2 of them (8\%) its insertion interdigitated with the contralateral. In $5(16.67 \%)$ cases, 3 female $(60 \%)$ and 2 male (40\%), the absence of that ligament was found. The reflected ligament was identified in most of our specimens. Being part of the posterior limit of the superficial inguinal ring, its anatomical knowledge is important for the surgical repair of inguinal hernias with prosthetic placement, using anterior access.

KEY WORDS: Reflected ligament; Inguinal region; External oblique muscle; Línea alba.

\section{REFERENCIAS BIBLIOGRÁFICAS}

Barroetaveña, J.; Herszage, L.; Barroetaveña, J. L. \& Ainstein, R. Hernias de la ingle. $3^{\mathrm{a}}$ ed. Buenos Aires, El Ateneo, 1993.

Carbonell Tatay, F. Hernia Inguinocrural. Madrid, Ethicon, 2001.

Casiraghi, J. C.; Anapios, R. \& Cúneo, J. L. Anatomía del cuerpo humano: funcional y quirúrgica. Buenos Aires, El Ateneo, 1969.

Drake, R. L.; Vogl, W. \& Mitchell, A. W. M. Gray anatomía para estudiantes. Madrid, Elsevier, 2005.

Ferraina, P. \& Oria, A. Cirugía de Michans. 5a ed. Buenos Aires, El Ateneo, 2002.

Latarjet, M. \& Ruiz Liard, A. Anatomía Humana. $3^{\text {a }}$ ed. Buenos Aires, Médica Panamericana, 2005.

Lytle, W. J. Inguinal anatomy. J. Anat., 128(Pt. 3):581-94, 1979.

Lytle, W. J. The inguinal and lacunar ligaments. J. Anat., 118(Pt. 2):241-51, 1974.

Moore, K. L.; Dalley, A. F. \& Agur, A. M. Moore Anatomía con Orientación Clínica. $7^{\mathrm{a}}$ ed. Barcelona, Lippincott Williams \& Wilkins, 2013.

Nyhus, L. M. \& Condon, R. E. Hernia. 4a ed. Philadelphia, J. B. Lippincott, 1995.

Pró, E. A. Anatomía Clínica. $2^{\text {a }}$ ed. Buenos Aires, Médica Panamericana, 2014.

Tubbs, R. S.; McDaniel, J. G.; Burns, A. M.; Kumbla, A.; Cossey, T. D.; Apaydin, N.; Comert, A.; Acar, H. I.; Tekdemir, I.; Shoja, M. M. \& Loukas. M. Anatomy of the reflected ligament of the inguinal region. Rom. J. Morphol. Embryol., 50(4):689-91, 2009.

Rouvière, H. \& Delmas, A. Anatomía Humana: Descriptiva, Topográfica y Funcional. 11 a ed. Barcelona, Masson, 2005.

Testut, L. \& Jacob, O. Tratado de anatomía topográfica con aplicaciones médicoquirúrgicas. 8ª ed. Barcelona, Salvat Editores, 1964.

Testut, L. \& Latarjet, A. Tratado de Anatomía Humana. $9^{\mathrm{a}}$ ed. Barcelona, Salvat, 1984

Dirección para Correspondencia:

Rubén Daniel Algieri

Especialista en Cirugía General. Jefe de Servicio de Cirugía General del Hospital Aeronáutico Central. Prof. Regular Adjunto de Anatomía y Docente Adscripto en Cirugía

III Cátedra de Anatomía Humana

Facultad de Medicina

Universidad de Buenos Aires

Paraguay 2155. C.A.B.A.

Buenos Aires

ARGENTINA

Email: rdalgieri08@hotmail.com

Recibido : 16-01-2015 Aceptado: 25-09-2015 